\title{
Metal-Free Conversion of Carboxamides to Ureas Using Tertiary Amines and Iodosylmesitylene ${ }^{\dagger}$
}

\author{
Hoon Han and Sukbok Chang* \\ Department of Chemistry and Molecular-Level Interface Research Center, Korea Advanced Institute of Science and \\ Technology (KAIST), Daejon 305-701, Korea. E-mail: sbchang@kaist.ac.kr \\ Received November 25, 2009, Accepted February 2, 2010
}

Key Words: Ureas, Amides, Curtius rearrangement, $N$-Dealkylaton, Iodosylmesitylene

Ureas are recognized as an important synthetic building unit, ${ }^{1}$ finding a wide range of applications in the preparation of agrochemicals, ${ }^{2}$ petrochemicals, ${ }^{3}$ and pharmaceuticals. ${ }^{4}$ In addition, hydrogen bond-mediated communication between ureas and certain types of compounds is an important structural motif in asymmetric catalysis, ${ }^{5}$ molecular recognition, ${ }^{6}$ and crystal engineering. ${ }^{7}$ Although numerous procedures have been developed for the urea synthesis, the most commonly employed strategy involves the reaction of amine precursors with phosgene, ${ }^{8}$ or its surrogates, ${ }^{9}$ isocyanates, ${ }^{10}$ or carbamates. ${ }^{11}$ Whereas these traditional methods are operative under conventional reaction conditions, they often lead to incomplete conversion. ${ }^{12}$ Additional drawback of these protocols is that unsymmetric ureas are difficult to prepare in many cases.

In addition to the above mentioned stoichiometric procedures, preparation of ureas via an oxidative carbonylation of amines has also been extensively investigated using various transition metal species such as Pd, ${ }^{13} \mathrm{Ru},{ }^{14} \mathrm{Co},{ }^{15} \mathrm{Mn},{ }^{16} \mathrm{Se},{ }^{17}$ or W. ${ }^{18} \mathrm{Al}-$ though the type of catalyst choice has been significantly expanded in recent years, some limitations still remain to be overcome such as the requirement of high pressure $\mathrm{CO}$ or harsh reaction conditions. During the course of our recent studies on the nitrogen group transfer, ${ }^{19}$ we unexpectedly found that ureas were obtained upon the reaction of carboxamides with tertiary amines under oxidative conditions (Scheme 1). The transformation is interesting especially due to the fact that ureas are produced by involving a path of $N$-dealkylation of the employed tertiary amines. ${ }^{20}$ Herein, we describe a facile preparation of unsymmetric ureas from the oxidative reaction of amides with tertiary amines, and a mechanistic proposal is also presented in which the Curtius rearrangement is involved to generate isocyanate intermediate in situ.

We first tried to optimize the reaction variables such as type of working amides, amines, oxidants, solvents, and temperatures using some representative carboxamides and triethylamine as test substrates (Table 1). With the employment of iodosylmesitylene as a stoichiometric oxidant, the reaction turned out to be quite sensitive to the choice of amides examined. For instance, when benzamide was allowed to react with triethylamine in benzene, $N, N$-diethyl- $N$ '-phenylurea was obtained only in low yield (entry 1). In a sharp contrast, a reaction of 2-pyridinecarboxamide afforded the corresponding urea in excellent yield

${ }^{\dagger}$ This paper is dedicated to Professor Sunggak Kim on the occasion of his honorable retirement. under the same reaction conditions (entry 2).

This result led us to envision a chelating effect on the reaction efficiency. ${ }^{21}$ To test this hypothesis, when we employed nicotinamide and isonicotinamide as substrates, to our surprise, the reactions were found to proceed to afford moderate yields (entries 3 and 4, respectively), implying that there is no significant chelation effects to dictate the reaction efficiency. Rather it was proposed that electronic effects are more important to achieve a high reaction profile. In fact, that hypothesis became more evident when 4-nitrobenzamide was reacted with triethylamine under the conditions to provide the desired product in acceptable yield (compare entries 1 and 5). In addition, reactions of benzeneacetamide or 2-pyridineacetamide gave the corresponding ureas in low yields (entries 6 and 7, respectively).

It should be mentioned that the transformation was best performed with the use of iodosylmesitylene as an oxidant. In fact, when other oxidants were used instead, much lower product yields were achieved. For example, whereas 87\% NMR yield of $N, N$-diethyl- $N$ '-(2-pyridyl)urea was obtained using iodosylmesitylene (entry 2), the replacement of the oxidant with iodobenzenediacetate or iodosylbenzene provided less than $10 \%$ yields under otherwise identical conditions.

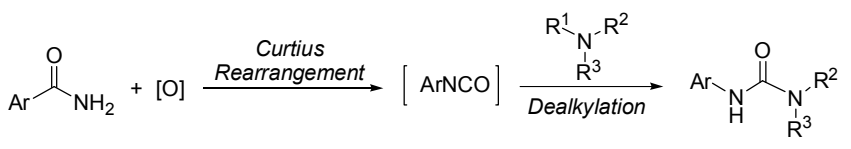

Scheme 1

Table 1. Optimization of the dealkylative urea formation reaction

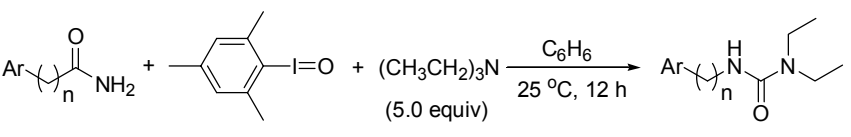
(2.5 equiv)

\begin{tabular}{clcc}
\hline entry & $\mathrm{Ar}$ & $\mathrm{n}$ & ${\text { yield }(\%)^{a}}^{a}$ \\
\hline 1 & $\mathrm{C}_{6} \mathrm{H}_{5}$ & 0 & 20 \\
2 & 2-Pyridyl & 0 & 87 \\
3 & 3-Pyridyl & 0 & 56 \\
4 & 4-Pyridyl & 0 & 64 \\
5 & $\left(4-\mathrm{NO}_{2}\right) \mathrm{C}_{6} \mathrm{H}_{4}$ & 0 & 65 \\
6 & $\mathrm{C}_{6} \mathrm{H}_{5}$ & 1 & 36 \\
7 & 2-Pyridyl & 1 & 32 \\
\hline
\end{tabular}

${ }^{a}$ H-NMR yield using anisole as an internal standard. 
Table 2. Dealkylative oxidative formation of unsymmetric ureas

\begin{tabular}{|c|c|c|c|c|}
\hline$\prod_{0}^{r}$ & (2.5 equiv) & quiv) & $\frac{\mathrm{C}_{6} \mathrm{H}_{6}}{25^{\circ} \mathrm{C}, 1 \mathrm{~h}}$ & 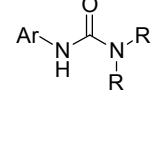 \\
\hline entry & amide (Ar) & $\mathrm{R}$ & & yield $(\%)^{a}$ \\
\hline 1 & 2-Py (1) & $\mathrm{CH}_{3} \mathrm{C}$ & $\mathrm{CH}_{2-}$ & 80 \\
\hline 2 & 1 & $\mathrm{CH}_{3} \mathrm{C}$ & $\left.\mathrm{CH}_{2}\right)_{2-}$ & 53 \\
\hline 3 & 1 & $\mathrm{CH}_{3} \mathrm{C}$ & $\left.\mathrm{CH}_{2}\right)_{3-}$ & 45 \\
\hline 4 & 1 & $\mathrm{CH}_{3}$ & $\left(\mathrm{CH}_{2}\right)_{4^{-}}$ & 68 \\
\hline 5 & 1 & $\mathrm{CH}_{2}=$ & $=\mathrm{CHCH}_{2}-$ & 54 \\
\hline 6 & 1 & $\mathrm{C}_{6} \mathrm{H}_{5}$ & $\mathrm{CH}_{2-}$ & 46 \\
\hline 7 & 1 & $\mathrm{HC} \equiv$ & $\mathrm{CCH}_{2-}$ & 20 \\
\hline 8 & $\left(4-\mathrm{NO}_{2}\right) \mathrm{C}_{6} \mathrm{H}_{4}(2)$ & $\mathrm{CH}_{3} \mathrm{C}$ & $\mathrm{CH}_{2-}$ & 50 \\
\hline 9 & 2 & $\mathrm{CH}_{3} \mathrm{C}$ & $\left.\mathrm{CH}_{2}\right)_{2-}$ & 57 \\
\hline 10 & 2 & $\mathrm{CH}_{3} \mathrm{C}$ & $\left(\mathrm{CH}_{2}\right)_{3-}$ & 53 \\
\hline 11 & 2 & $\mathrm{CH}_{3} \mathrm{C}$ & $\left(\mathrm{CH}_{2}\right)_{4^{-}}$ & 60 \\
\hline 12 & 2 & $\mathrm{CH}_{2}=$ & $=\mathrm{CHCH}_{2}-$ & 65 \\
\hline 13 & 2 & $\mathrm{C}_{6} \mathrm{H}_{5}$ & $\mathrm{CH}_{2-}^{-}$ & 41 \\
\hline 14 & 2 & $\mathrm{HC} \equiv$ & $\mathrm{CCH}_{2-}$ & 25 \\
\hline
\end{tabular}

${ }^{a}$ Isolated yield.

Table 3. Dealkylative urea formation using differently substituted tertiary amines

$$
\text { entry equiv) }
$$

${ }^{a}$ Isolated yield.

Under the above optimized conditions, a wide range of tertiary amines were employed to react with either picolineamide (1) or 4-nitrobenzamide (2) and the results are presented in Table 2. In general, the reactions took place smoothly to afford the desired unsymmetric ureas in moderate to good yields.
Path A

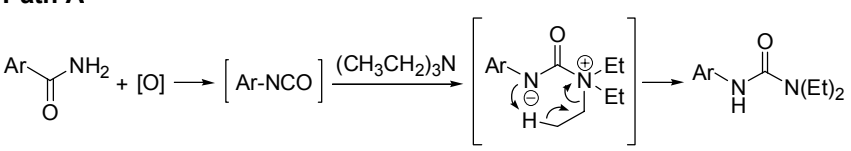

Path B

$\left(\mathrm{CH}_{3} \mathrm{CH}_{2}\right)_{3} \mathrm{~N} \stackrel{[\mathrm{O}]}{\longrightarrow}\left[\left(\mathrm{CH}_{3} \mathrm{CH}_{2}\right) \mathrm{N}-\mathrm{O} \longrightarrow\left(\mathrm{CH}_{3} \mathrm{CH}_{2}\right)_{2} \mathrm{NH}\right] \stackrel{[\mathrm{Ar}-\mathrm{NCO}]}{\longrightarrow} \mathrm{Ar}-\mathrm{N}_{\mathrm{H}} \stackrel{\mathrm{O}}{\mathrm{N}} \underset{\mathrm{N}(\mathrm{Et})_{2}}{ }$

Scheme 2

While tertiary amines of simple aliphatic chains such as ethyl-, propyl-, butyl-, or pentyl groups were all efficiently dealkylated under the reaction conditions to give the corresponding $N, N$ dialkylated ureas upon the reaction with either 1 or $\mathbf{2}$ (entries $1-4$, and 8-11, respectively).

Quite surprisingly, different types of tertiary amines other than simple trialkylamines such as triallylamine, tribenzylamine, or tripropargylamine were also found to readily participate in the reaction (entries 5-7 and 12-14, respectively) although the product yields were generally lower than those with aliphatic trialkylamines.

We subsequently tried to observe a possibility of regioselective dealkylative urea formation with the use of tertiary amines having different substituents (Table 3 ). When $N, N$-diethylcyclohexylamine was allowed to react with picolinamide (1) under the oxidative conditions, a mixture of two ureas are obtained in a moderate combined yield (entry 1 ). While the major product (3) was resulted from the dealkylation of an ethyl group, dealkylation of a cyclohexyl moiety turned out to be a minor path leading to $\mathbf{4}$ in only poor yield. A similar trend of favored dealkylation of an ethyl group over a bulkier isopropyl substituent was also observed in the reaction of $N, N$-diisopropylethylamine (entry 2). It was highly interesting to see that a similar degree of dealkylation between two types of substituents took place when diethylaminoacetonitrile was subjected to the reaction conditions (entry 3 ). In addition, no significant difference of regioselectivity was observed in the reaction with diethylallylamine (entry 4).

When 2-picolinamide was treated with iodosylmesitylene in the presence of an alcohol instead of tertiary amines, the corresponding carbamate $\mathbf{1 1}$ was obtained in moderate yield (Eq 1), implying that the transformation indeed proceeds via an isocyanate intermediate through the Curtius rearrangement. ${ }^{22}$ Although the exact mechanism is not clear at this stage, it can be proposed that tertiary amines attack the in situ generated isocyanate to form a zwitterionic species, in which an intramolecular hydrogen abstraction is assumed to take place subsequently (Scheme 2, Path A). ${ }^{23}$ It is believed that release of an olefin side product may accelerate the progress of the reaction pathways. However, other mechanistic possibilities have to be also considered especially for amines that do not have a $\beta$ hydrogen. For instance, oxidation of tertiary amines may be envisioned to take place by the action of the employed oxidants to afford to their corresponding $N$-oxides which then undergo a dealkylation leading to secondary amines to react with isocyanates (Scheme 2, Path B). ${ }^{24}$ 


$$
\underbrace{\mathrm{Nos}}_{\substack{\mathrm{MeOH} / \mathrm{C}_{6} \mathrm{H}_{6} \\ 25^{\circ} \mathrm{C}, 1 \mathrm{~h}}}
$$

In conclusion, a new synthetic route has been developed for the preparation of ureas from the reaction of carboxamides with tertiary amines with the use of iodosylmesitylene as a stoichiometric oxidant. Although the reaction proceeds under the mild conditions to afford urea products in moderate yields, further optimization studies are required to improve the reaction efficiency and regioselectivity in the dealkylative pathway.

\section{Experimental Section}

General procedure. A mixture of picolinamide $(31.0 \mathrm{mg}$, $0.25 \mathrm{mmol}$ ), iodosylmesitylene ( $164 \mathrm{mg}, 0.625 \mathrm{mmol}$ ), and triethylamine $(174 \mu \mathrm{L}, 1.25 \mathrm{mmol})$ in benzene $(2 \mathrm{~mL})$ was stirred at $25{ }^{\circ} \mathrm{C}$ for $1 \mathrm{~h}$ under $\mathrm{N}_{2}$. After the reaction was completed, the mixture was concentrated in vacuo. The crude material was purified by silica gel flash column chromatography (EtOAc/Hx, 1:2) to give $N, N$-diethyl- $N$ '-(2-pyridyl)urea as pale yellow oil; ${ }^{1} \mathrm{H}$ NMR $\left(300 \mathrm{MHz}, \mathrm{CDCl}_{3}\right) \delta 1.24(\mathrm{t}, J=6.5 \mathrm{~Hz}, 6 \mathrm{H}), 3.39(\mathrm{q}$, $J=7.2 \mathrm{~Hz}, 1 \mathrm{H}), 6.92(\mathrm{~m}, 1 \mathrm{H}), 7.27$ (br, $1 \mathrm{H}), 7.63(\mathrm{~m}, 1 \mathrm{H}), 8.10$ $(\mathrm{d}, J=8.6 \mathrm{~Hz}, 1 \mathrm{H}), 8.19(\mathrm{~m}, 1 \mathrm{H}) ;{ }^{13} \mathrm{C} \mathrm{NMR}\left(75 \mathrm{MHz}, \mathrm{CDCl}_{3}\right)$ $\delta 13.8,41.3,113.2,118.1,138.0,147.3,147.4,152.9,153.7$; IR (neat) v 2874, 2361, 2341, 1869, 1734, 1540, 1431, $1296 \mathrm{~cm}^{-1}$; HRMS (FAB) $m / z$ calcd. for $\mathrm{C}_{13} \mathrm{H}_{12} \mathrm{~N}_{2}[M+H]^{+} 194.1293$, found 194.1292.

Acknowledgments. This research was supported by the Korea Research Foundation Grant (KRF-2008-C00024, Star Faculty Program).

\section{References}

1. (a) Tafesh, A. M.; Weiguny, J. Chem. Rev. 1996, 96, 2035. (b) Spanu, P.; Ulgheri, F. Curr. Org. Chem. 2008, 12, 1071.

2. Zakrzewski, J.; Krawczyk, M. Heteroatom Chem. 2006, 17, 393.

3. Klingstedt, F.; Arve, K.; Eränen, K.; Murzin, D. Y. Acc. Chem. Res. 2006, 39, 273.

4. (a) Das, J.; Lin, J.; Moquin, R. V.; Shen, Z.; Spergel, S. H.; Wityak, J.; Doweyko, A. M.; DeFex, H. F.; Fang, Q.; Pang, S.; Pitt, S.; Shen, D. R.; Schieven, G. L.; Barrish, J. C. Bioorg. Med. Chem. Lett. 2003, 13, 2145. (b) Kurihara, M.; Rouf, A. S. S.; Kansui, H.; Kagechika, H.; Okuda, H.; Miyata, N. Bioorg. Med. Chem. Lett. 2004, 14, 4131.

5. (a) Christianson, D. W. Acc. Chem. Res. 2005, 38, 191. (b) Doyle, A. J.; Jacobsen, E. N. Chem. Rev. 2007, 107, 5713. (c) Zhang, Z.; Schreiner, P. R. Chem. Soc. Rev. 2009, 38, 1187.

6. (a) Bell, T. W.; Hext, N. M. Chem. Soc. Rev. 2004, 33, 589. (b) Amendola, V.; Esteban-Gómez, D.; Fabbrizzi, L.; Licchelli, M.
Acc. Chem. Res. 2006, 39, 343.

7. Borovik, A. S. Acc. Chem. Res. 2005, 38, 54.

8. Petersen, U. In Methoden der Organischen Chemie: Houben-Weyl; E4; G. Thieme Verlag: New York, 1983; pp 334.

9. Majer, P.; Randad, R. S. J. Org. Chem. 1994, 59, 1937.

10. (a) Barrett, A. G. M.; Betts, M. J.; Fenwick, A. J. Org. Chem. 1985, 50, 169. (b) Wang, Y.; Wheelhouse, R. T.; Zhao, L.; Langnel, D. A. F.; Stevens, M. F. G. J. Chem. Soc., Perkin Trans. 1 1998, 1669.

11. (a) Basha, A. Tetrahedron Lett. 1988, 29, 2525. (b) Matsumura, Y.; Satoh, Y.; Onomura, O.; Maki, T. J. Org. Chem. 2000, 65, 1549.

12. Aguirre, I. de; Collot, J. Bull. Soc. Chim. Belg. 1989, 98, 19.

13. (a) Grupte, S. P.; Chaudhari, R. V. J. Catal. 1988, 114, 246. (b) Shi, F.; Deng, Y.; SiMa, T.; Yang, H. Tetrahedron Lett. 2001, 42, 2161. (c) Gabriele, B.; Salerno, G.; Mancuso, R.; Costa, M. J. Org. Chem. 2004, 69, 4741.

14. Mulla, S. A. R.; Rode, C. V.; Kelkar, A. A.; Gupte, S. P. J. Mol. Catal. A. Chem. 1997, 122, 103.

15. Bassoli, A.; Rindone, B.; Tollari, S.; Chioccara, F. J. Mol. Catal. 1990, 60, 41 .

16. Dombek, B. D.; Angelici, R. J. J. Organomet. Chem. 1977, 134, 203.

17. (a) Sonoda, N.; Yasuhara, T.; Kondo, K.; Ikeda, T.; Tsutsumi, S. J. Am. Chem. Soc. 1971, 93, 6344. (b) Yoshida, T.; Kambe, N.; Murai, S.; Sonoda, N. Tetrahedron Lett. 1986, 27, 2037. (c) Sonoda, N. Pure Appl. Chem. 1993, 65, 699.

18. (a) McCusker, J. E.; Main, A. D.; Johnson, K. S.; Grasso, C. A.; McElwee-White, L. J. Org. Chem. 2000, 65, 5216. (b) McCusker, J. E.; Qian, F.; McElwee-White, L. J. Mol. Catal. A-Chem. 2000, $159,11$.

19. (a) Han, H.; Bae, I.; Yoo, E. J.; Lee, J.; Do, Y.; Chang, S. Org. Lett. 2004, 6, 4109. (b) Bae, I.; Han, H.; Chang, S. J. Am. Chem. Soc. 2005, 127, 2038. (c) Cho. S. H.; Yoo, E. J.; Bae, I.; Chang. S. J. Am. Chem. Soc. 2005, 127, 16046. (d) Yoo. E. J.; Bae, I.; Cho, S. H.; Han, H.; Chang, S. Org. Lett. 2006, 8, 1347. (e) Chang, S.; Lee, M. J.; Jung, D. Y.; Yoo, E. J.; Cho, S. H.; Han, S. K. J. Am. Chem. Soc. 2006, 128, 12366. (f) Han, H.; Park, S. B.; Kim, S. K.; Chang. S. J. Org. Chem. 2008, 73, 2862.

20. (a) Brewster, J. H.; Eliel, E. L. In Organic Reactions; Adams, R., Ed.; John Wiley and Sons: New York, 1953; Vol. VII, pp 142. (b) Olofoson, R. A.; Schur, R. C.; Bunes, L.; Pepe, J. P. Tetrahedron Lett. 1977, 18, 1567. (c) Cooley, J. H.; Evain, E. J. Synthesis $1989,1$.

21. For related works on the chelation-driven reactions from this laboratory, see: (a) Ko, S.; Na, Y.; Chang, S. J. Am. Chem. Soc. 2002, 124, 750. (b) Ko, S.; Lee, C.; Choi, M.-G.; Na, Y.; Chang, S. J. Org. Chem. 2003, 68, 1607. (c) Ko, S.; Han, H.; Chang, S. Org. Lett. 2003, 5, 2687. (d) Na, Y.; Ko, S.; Hwang, L. K.; Chang, S. Tetrahedron Lett. 2003, 44, 4475. (e) Ko, S.; Kang, B.; Chang, S. Angew. Chem., Int. Ed. 2005, 44, 455. (f) Park, E. J.; Lee, J. M.; Han, H.; Chang, S. Org. Lett. 2006, 8, 4355.

22. Trost, B. M. In Comprehensive Oganic Synthesis; Pergamon: Oxford, 1991; pp 795.

23. (a) Abdul Hai, S. M.; Perveen, S.; Khan, R. A.; Khan, K. M.; Afza, N. Nat. Prod. Res. 2003, 17, 351. (b) Aranha, R. M.; Bowser, A. M.; Madalengoitia, J. S. Org. Lett. 2009, 11, 575.

24. (a) Okazaki, R.; Tokitoh, N. J. Chem. Soc., Chem. Commun. 1984, 192. (b) Monkovic, I.; Wong, H.; Bachand, C. Synthesis 1985, 770. 